International Journal of Physical Research, 5(2)(2017) $101-103$
International Journal of Physical Research
SPC
Website: www.sciencepubco.com/index.php/IJPR
doi: $10.14419 /$ ijpr.v5i2.8396
Research paper

\title{
New study of geometry effect on chiral metamaterial nanostructures
}

\author{
Mezache Zinelabiddine ${ }^{1} *$, Zara Chemseddine ${ }^{2}$, Larioui Fateh ${ }^{2}$, Benabdelaziz Fatiha ${ }^{2}$ \\ ${ }^{1}$ Institue of Optics and fine mechanics, University of Ferhat Abbas Setif 1, 19000 Setif, Algeria \\ ${ }^{2}$ Department of Electronic, University of Brothers Mentouri Constantine 1, 25000 Constantine, Algeria \\ *Corresponding author E-mail: zinemezaache@yahoo.fr
}

\begin{abstract}
In this paper, the Comsol Multiphysics version 5.0 is used to study the effect of geometric parameters on transmission of chiral metamaterial nanostructures. The angle of the chiral metamaterial element was varied to see his effect. Transmission coefficient $\left(S_{21}\right)$ and reflection coefficient $\left(\mathrm{S}_{11}\right)$ are computed. In the case of nano chiral metamaterial structure, and depending on the application, the electromagnetic behavior can be adjusted by changing the angle of the chiral element.
\end{abstract}

Keywords: Chiral Metamaterial; Nanostructures.

\section{Introduction}

Chiral metamaterial materials have attracted great interest in the breast of the scientific community. This type of medium to an interesting phenomenon is negative reflection (specific property for metamaterial). In addition, the artificial composite of open structures is a new perspective in the reflection and transmission effects of an electromagnetic wave. Today, there are several theoretical studies of modeling, simulation, and experimental work on these new materials [1-6]. In this paper, we will simulate a nanostructure of chiral metamaterial (FIG. 1), representing both the properties of metamaterial and chirality (optical rotation and circular dichroism), from the determination of the parameter's $S$, Which are not identical for the chiral medium due to the existence of circular polarization of left and right light (LCP and RCP). The chiral metamaterial cell (FIG. 1) is considered in a lossless air block, and excited by a polychromatic radiation source with the circularly polarized left LCP wave, and also for the right-hand RCP wave. The frequency varies from infrared $350 \mathrm{THz}$ to ultraviolet $950 \mathrm{THz}$. The angel $\theta$ Defined in (FIG. 1) has been varied according to the values $90^{\circ}$ and $60^{\circ}$, and then we have shown the angle effect on chiral metamaterial nanostructures.

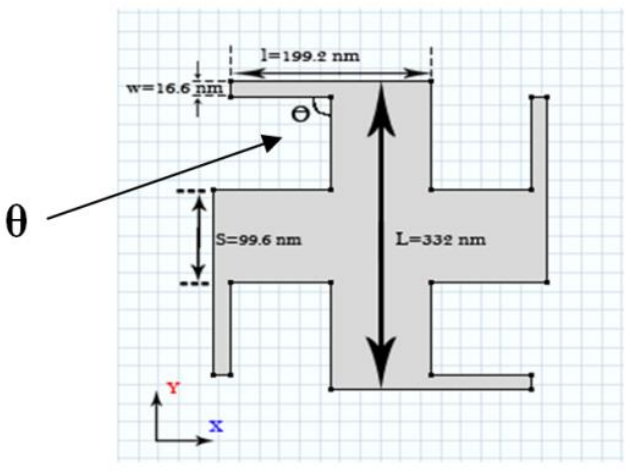

Fig. 1: Representation of the Chiral Metamaterials Nanostructure [7].

\section{Results and comments}

In this stage of our work we have studied two arrays $(2 \times 2)$ of cell elements, presented below (FIG. 2, 3). The wave propagated respectively in the two arrays is directed along the $\mathrm{Z}$ axis, for the two cases of left circular polarization LCP and right RCP. The thicknesses of the gold $(\mathrm{Au})$ layers are identical: $\mathrm{C}_{1}=\mathrm{C}_{3}=48 \mathrm{~nm}$, and the $\mathrm{Al}_{2} \mathrm{O}_{3}$ layer has a thickness of: $\mathrm{C}_{2}=24 \mathrm{~nm}$.

$\mathrm{S}_{11}$ : Input reflection coefficient, when the output is adapted. $\mathrm{S}_{21}$ : Direct transmission coefficient, when the output is adapted. 

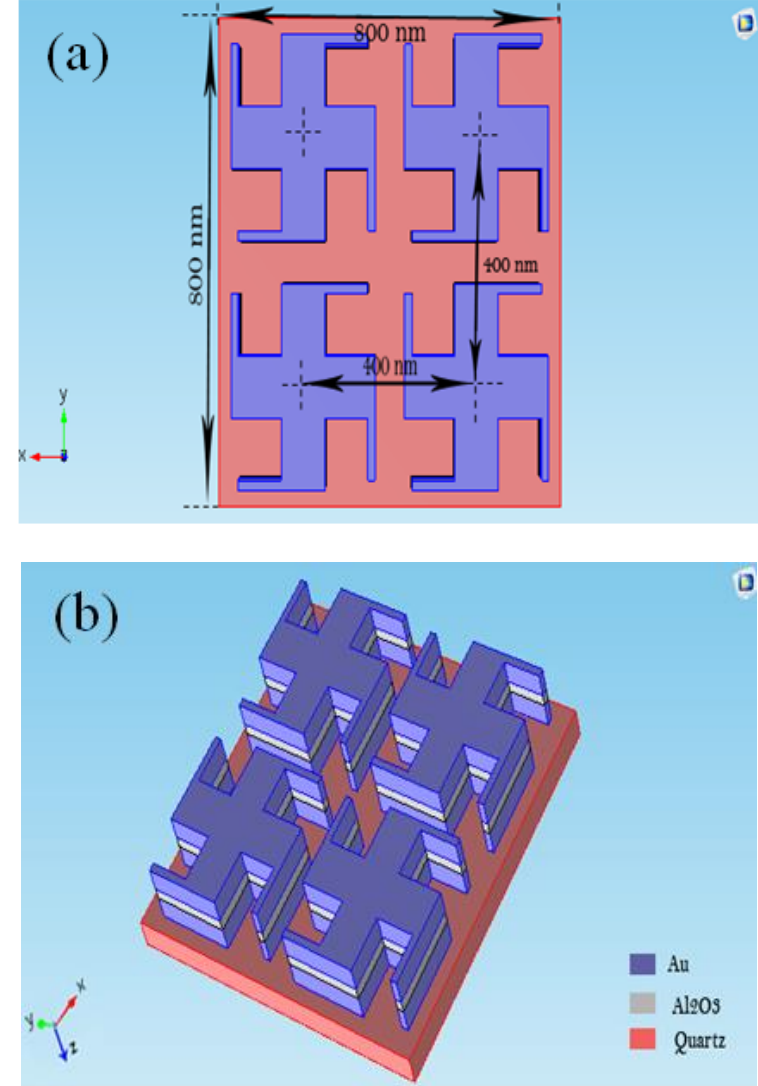

Fig. 2: (A) - Top View for Array of Elements with $\Theta=90^{\circ}$ According to the Plane (X, Y), (B)-3D Representation for Array of Elements with $\Theta=$ $90^{\circ}$.
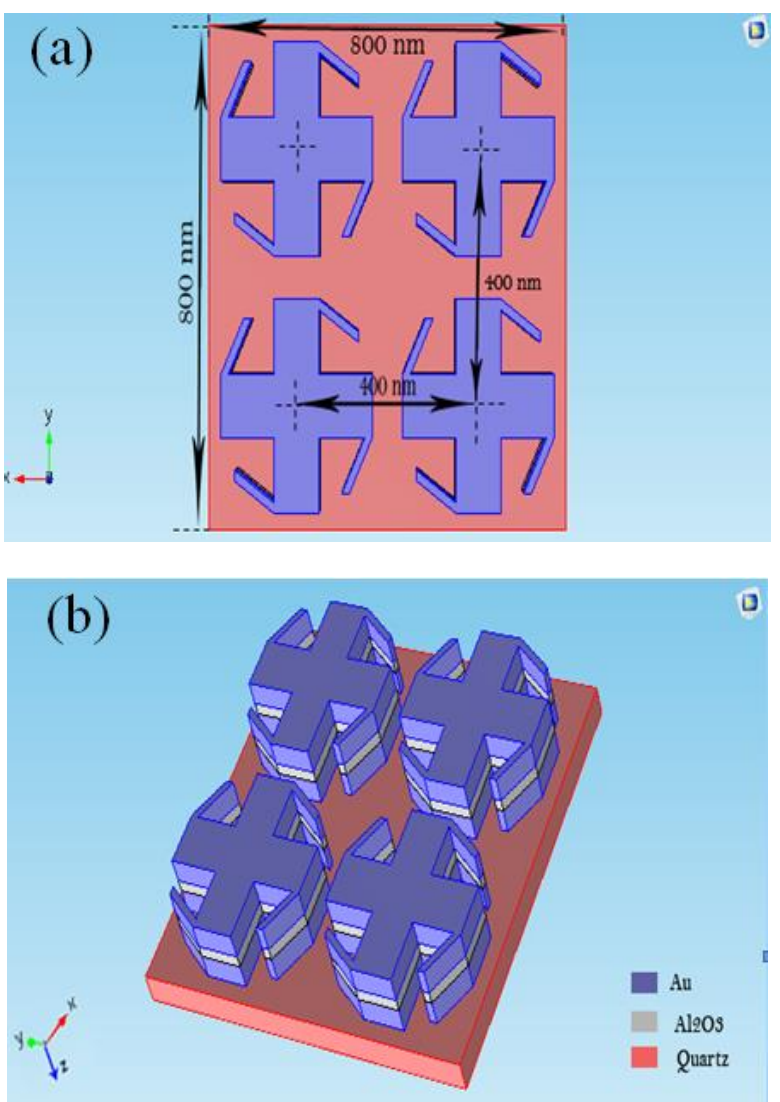

Fig. 3: (A) - Top View for Array of Elements with $\Theta=60^{\circ}$ According to the Plane (X, Y), (B)-3D Representation for Array of Elements with $\Theta=$ $60^{\circ}$.

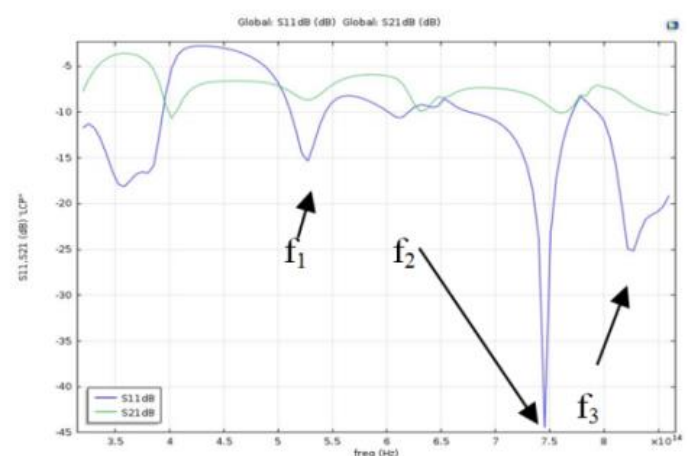

(a)

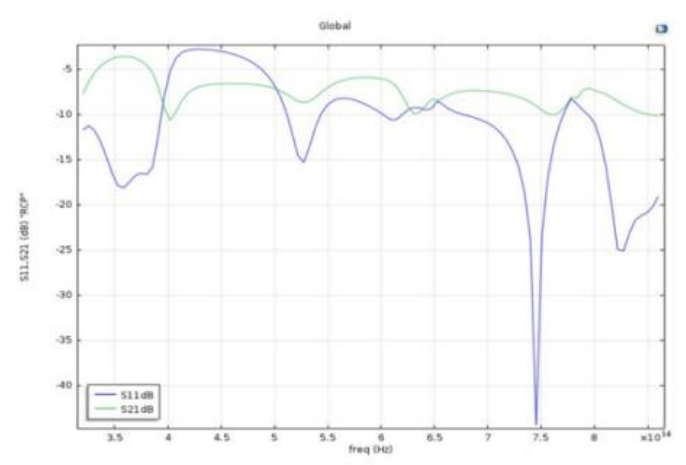

(b)

Fig. 4: (A) -Parameters $S_{11}, S_{21}$ LCP for Array of Element with $\Theta=90^{\circ}$, (B) - Parameters $S_{11}, S_{21}$ RCP for Array of Element with $\Theta=90^{\circ}$.

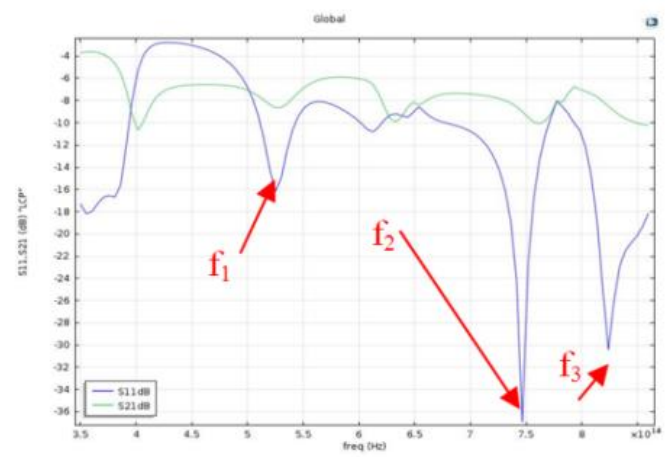

(a)

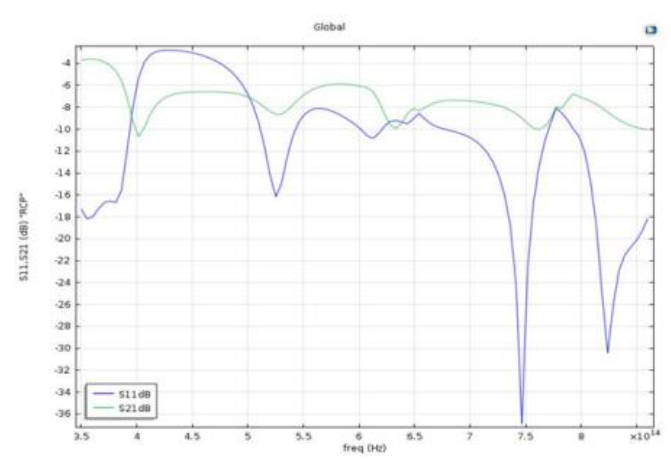

(b)

Fig. 5: (A) -Parameters $S_{11}, S_{21}$ LCP for Array of Element with $\Theta=60^{\circ}$, (B) - Parameters $S_{11}, S_{21}$ RCP for Array of Element with $\Theta=60^{\circ}$.

The curves presenting the reflection coefficients, respectively relative to the LCP and RCP waves (FIG. 4), for the array of an element with $\theta=90^{\circ}$ (FIG. 2), simulated by Comsol Multiphysics 5.0, The values in $\mathrm{dB}$ of the parameters, $\mathrm{S}$ is apparent at frequencies $f_{1}, f_{2}$ and $f_{3}$, with the presence of certain harmful perturbations 
and have a remarkable peak at the frequency $\mathrm{f}_{2}=745.454 \mathrm{THz}$ for $\mathrm{LCP}$ and $745.455 \mathrm{THz}$ for RCP, respectively relating to frequency bands linked according to the types of applications we need. Indeed, for array of an element with $\theta=60^{\circ}$ (FIG. 3), it is maintained identical to the previous case, so the peak of the curve of the reflection parameter is located at the value $\mathrm{f}_{2}=746.56 \mathrm{THz}$ for LCP and 746.665 THZ for RCP. According to the curves (FIG. 5) of the geometrically modified structure with $\theta=60^{\circ}$ are distinctly improved, and have clearly separated windows on the transmission of the structure for applications in well-defined frequency ranges. Consequently, the modified structure with $\theta=60^{\circ}$ implied an increase in the resonant frequency $\left(f_{1}, f_{2}, f_{3}\right)$ for RCP (the accelerated waves), counter to LCP (the decelerated waves), which illustrates and confirms the dichroism effect.

\section{Conclusion}

The results obtained by simulation; have led us to study the effect of geometric parameters such as the angle of the chiral element $(\theta)$ on the transmission coefficient $\left(S_{21}\right)$, the reflection coefficient $\left(S_{11}\right)$. We have highlighted this effect. Our perspectives on the subject of nanophotonics will be the study of physical properties for the application of optical antennas [6], and other applications, for example optical detection in biology [7].

\section{References}

[1] Akyurtlu, A., \& Werner, D. H. A novel dispersive FDTD formulation for modeling transient propagation in chiral metamaterials. IEEE Transactions on Antennas and Propagation, 2004; 52(9), 2267-2276. https://doi.org/10.1109/TAP.2004.834153.

[2] Wang, X., Werner, D. H., Li, L. W., \& Gan, Y. B. Interaction of electromagnetic waves with 3-D arbitrarily shaped homogeneous chiral targets in the presence of a lossy half space. IEEE Transactions on Antennas and Propagation, 2007; 55(12), 3647-3655. https://doi.org/10.1109/TAP.2007.910336.

[3] Kwon, D. H., Werner, P. L., \& Werner, D. H. Optical planar chira metamaterial designs for strong circular dichroism and polarization rotation.Optics express, 16(16), 2008; 11802-11807. https://doi.org/10.1364/OE.16.011802.

[4] Wang, B., Zhou, J., Koschny, T., Kafesaki, M., Soukoulis, C. M. Chiral Metamaterials: simulations and experiments. Journal of Optics A: Pure and Applied Optics, 2009; 11(11), 114003. https://doi.org/10.1088/1464-4258/11/11/114003.

[5] Soukoulis, C. M., Wegener, M. Past achievements and future chal lenges in the development of three-dimensional photonic Metamaterials. Nature Photonics, 2011; 5(9), 523-530. https://doi.org/10.1038/nphoton.2011.154.

[6] Mezache Z, Seghaouil S, Chaibi W, Benabdelaziz F, Optimization of Optical Nanoantenna Based on Structures Chiral Photonic Crystal, American Journal of Nanosciences. Vol. 3, No. 3, 2017, pp. $47-$ 52. doi: 10.11648/j.ajn.20170303.13

[7] Mezache, Z., Zara ch., Larioui, f., Benabdelaziz, f. Detection and analysis of human hemoglobin $\mathrm{HB}$ and $\mathrm{HBO} 2$ with new Nanosensor based on chiral metamaterials. International Journal of Physical Research, 2017; 5(2), 36-38. https://doi.org/10.14419/ijpr.v5i2.7780 